\title{
Gastric metastases from malignant melanoma
}

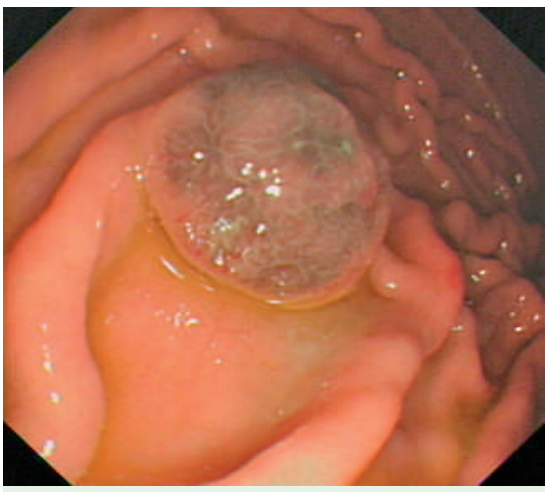

Fig. 1 Endoscopic view of one of the lesions in the gastric corpus.

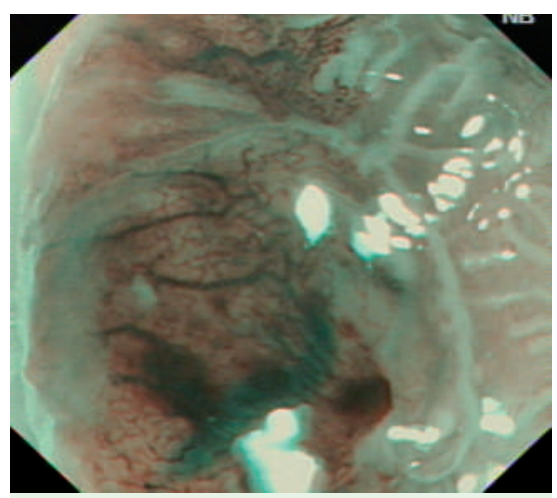

Fig. 2 The lesion shown in Fig. 1 visualized with narrow-band imaging in combination with magnification endoscopy.

A 44-year-old woman was referred to our hospital for investigation of epigastric pain. Her symptoms began 4 months before this hospitalization, with nausea and weight loss of $2 \mathrm{~kg}$; she denied having any other symptoms. Fourteen years earlier she had undergone surgery and immunotherapy for malignant melanoma in the face, but 10 years of follow-up investigations had been unremarkable. An outpatient endoscopy performed before she was admitted revealed "polyps in the gastric corpus," which were described as hemangiomas. On examination, she had moderate abdominal tenderness. The results of standard laboratory investigations were unremarkable.

Esophagogastroduodenoscopy in our department revealed three polypoid lesions in the gastric corpus, ranging in size from $6 \mathrm{~mm}$ to $12 \mathrm{~mm}$ ( Fig. 1). Narrow-band imaging of the lesions was performed

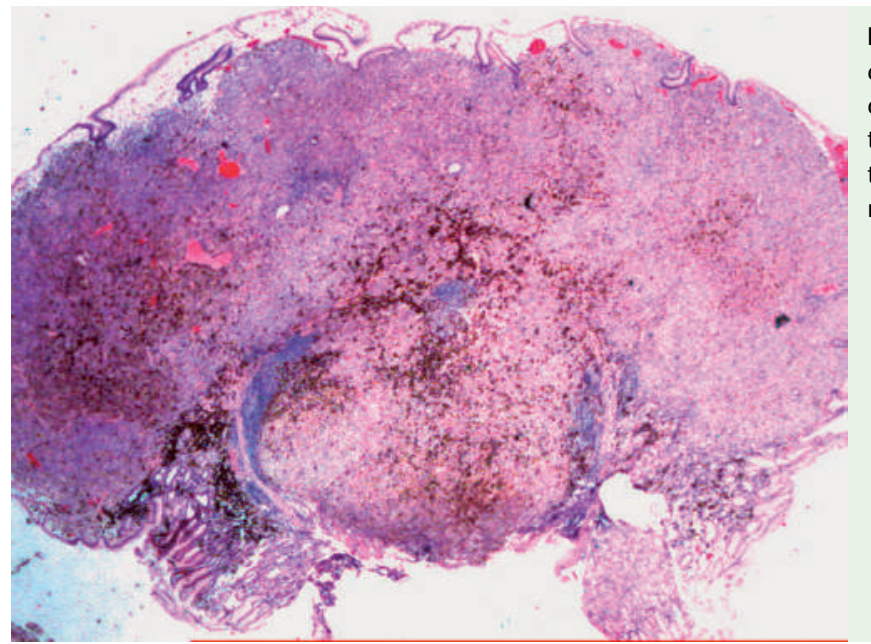

Fig. 3 Immunohistochemical examination of the biopsies revealed the lesions to be metastases of a malignant melanoma.

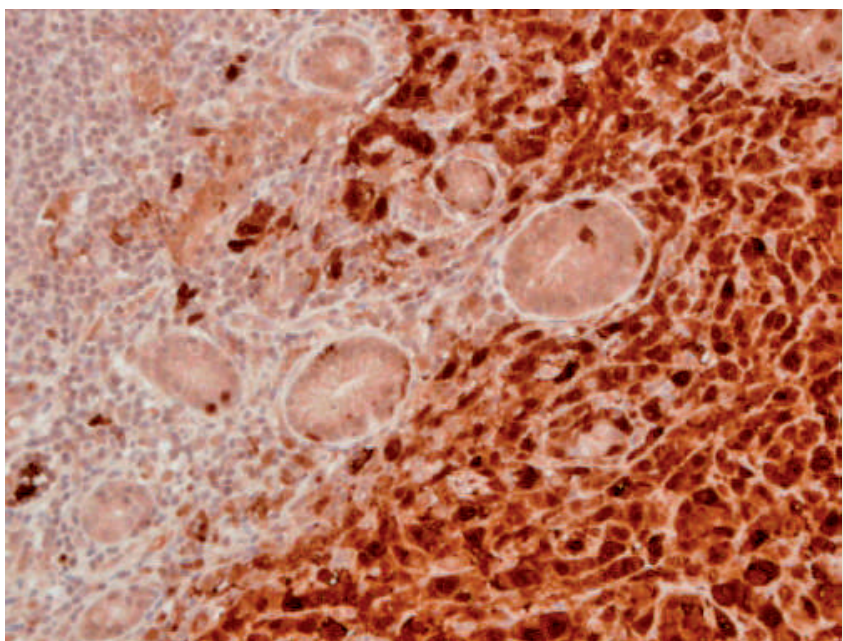

Fig. 4 Immunohistochemical staining was performed for vimentin, $\mathrm{HMB}-45$, and S-100.

( $\bullet$ Fig. 2) and two of these lesions were resected with a snare for histological examination ( Fig. 3,4).

Malignant melanoma is one of the most common malignancies to metastasize to the gastrointestinal tract. Metastases can occur at the time of primary diagnosis or decades later as the first sign of recurrence [1]. Symptoms can include abdominal pain, dysphagia, small-bowel obstruction, hematemesis, and melena. The prognosis is poor, with a median survival of 4-6 months [1]. Common sites for metastases of malignant melanoma in the gastrointestinal tract include the small bowel (50\%), colon (31.3\%) and anorec$\operatorname{tum}(25 \%)$ [2].

The case reported here shows that narrow-band imaging endoscopy can be helpful in the diagnosis of gastric mela- noma metastasis and is superior to standard white-light endoscopy alone. Narrow-band imaging endoscopy is able to differentiate pathological vessels from surrounding tissue using the part of visible light that is maximally absorbed by mucosal vessels. By using only blue and green light (i.e. without red light), details of the mucosal surface are picked out, giving a very clear image of the suspect lesion.

\section{Acknowledgment $\nabla$}

- Fig. 3 and $\mathbf{4}$ were reproduced with the kind permission of Dr. T. Wagner, Institute of Pathology, Klinikum Augsburg, Augsburg, Germany. 
Endoscopy_UCTN_Code_CCL_1AB_2AD_3AB

\section{Competing interests: None}

\section{B. Pommer, A. Probst, H. Messmann}

Department of Medicine III, Klinikum

Augsburg, Augsburg, Germany

\section{References}

1 Liang KV, Sanderson SO, Nowakowski GS, Arora AS. Metastatic malignant melanoma of the gastrointestinal tract. Mayo Clin Proc 2006; 81: $511-516$

2 Blecker D, Abraham S, Furth EE, Kochman ML. Melanoma in the gastrointestinal tract. Am J Gastroenterol 1999; 94: 3427-3433
Bibliography

DOI $10.1055 / \mathrm{s}-2007-995434$

Endoscopy 2008; 40: E30-E31

(c) Georg Thieme Verlag KG Stuttgart · New York .

ISSN 0013-726X

\section{Corresponding author}

\section{B. Pommer, MD}

Department of Medicine III

Klinikum Augsburg

Stenglinstrasse 2

86156 Augsburg

Germany

Fax: +49-821-400-3331

bjoern.pommer@klinikum-augsburg.de 